\title{
Wallenberg's Syndrome
}

National Institute of Neurological Disorders and Stroke (NINDS)

\section{Source}

National Institute of Neurological Disorders and Stroke (NINDS). Wallenberg's Syndrome

Information Page.

Wallenberg's syndrome is a neurological condition caused by a stroke in the vertebral or posterior inferior cerebellar artery of the brain stem. Symptoms include difficulties with swallowing, hoarseness, dizziness, nausea and vomiting, rapid involuntary movements of the eyes (nystagmus), and problems with balance and gait coordination. Some individuals will experience a lack of pain and temperature sensation on only one side of the face, or a pattern of symptoms on opposite sides of the body - such as paralysis or numbness in the right side of the face, with weak or numb limbs on the left side. Uncontrollable hiccups may also occur, and some individuals will lose their sense of taste on one side of the tongue, while preserving taste sensations on the other side. Some people with Wallenberg's syndrome report that the world seems to be tilted in an unsettling way, which makes it difficult to keep their balance when they walk. 